\title{
Crystal structure of InBi under pressure up to 75 GPa
}

\author{
V. F. Degtyareva \\ Institute of Solid State Physics, Russian Academy of Sciences, Chernogolovka, Moscow district, 142432 Russia \\ M. Winzenick and W. B. Holzapfel \\ FB 6 Physik, Universität-GH-Paderborn, 33095 Paderborn, Germany
}

(Received 1 July 1997)

\begin{abstract}
InBi is studied by energy-dispersive diffraction with synchrotron radiation in a diamond-anvil cell under pressure up to $75 \mathrm{GPa}$. Three phase transitions are observed from its initial tetragonal structure $t P 4$ with $c / a \approx 0.95$ to another $t P 4$ structure with $c / a \approx 0.65$ typical for the $\beta$-Np structure, than to a disordered tetragonal body-centered structure $t I 2$, and finally some hints are obtained for a transition to a cubic body-centered structure $c I 2$. All these transformations are reversible with large hysteresis. InBi( $t I 2)$, phase III, shows initially a steep increase in $c / a$ from 0.91 to 0.94 , in the pressure range from 20 to $30 \mathrm{GPa}$, and a nearly constant value of about 0.96 above $55 \mathrm{GPa}$. Hints for the possible stability of a new phase IV are noticed in the present experiments on decompression from $75 \mathrm{GPa}$ as admixture of new lines to phase III giving strong indications for a first-order phase transition with large hysteresis. The origin of the tetragonal distortion and the transformation to a cubic phase are discussed from the point of view of nesting the Fermi sphere in the Brillouin zone. [S0163-1829(98)00110-6]
\end{abstract}

\section{INTRODUCTION}

Structural systematics for the group IV elements and for the isoelectronic III-V compounds have been the subject of many recent experimental and theoretical high-pressure studies. Besides the increase in coordination numbers and packing efficiency a general structural sequence is found for $\mathrm{Si}$ and $\mathrm{Ge}$ of the type diamond-like $(c F 8) \rightarrow \beta$-tin(tI4) $\rightarrow$ simple hexagonal $(h P 1) \rightarrow h c p(h P 2) / f c c(c F 4) \quad$ experimentally $^{1-4}$ and in theoretical calculations. ${ }^{4-6}$

Isoelectronic III-V compounds display a similar structural sequence under pressure: zinc blende $(c F 8)$ $\rightarrow \mathrm{NaCl}(\mathrm{cF} 8) /$ white tin type $($ tI 4$) \rightarrow$ simple hexagonal $(h P 1)$. In addition to these rather simple structures also more complicated structures with free atom position parameters are found..$^{7-9}$ Besides disordered structures also structures with atomic ordering and large superlattices are observed, ${ }^{10-13}$ and theoretical studies have been performed for III-V compounds. ${ }^{14-18}$

However for the heavier III-V compound InBi experimental data were available up to now only for pressures below 3 GPa (Ref. 19) and a comparison with the heavier group IV element tin with its structural sequence $\beta$-tin(tI4) $\rightarrow b c t(t I 2) \rightarrow b c c(c I 2)$ (without $h P 1)^{20,22}$ leads to the expectation of a special behavior also for InBi.

At ambient conditions InBi forms a metallic compound with tetragonal structure of its own type, $t P 4$, space group $P 4 / \mathrm{nmm}$ with In in the special position $2 a(000 ; 1 / 21 / 20)$ and $\mathrm{Bi}$ in $2 c(01 / 2 z ; 1 / 20 \bar{z})$ with $z=0.393, a=500.0 \mathrm{pm}$, $c=477.3 \mathrm{pm}$, and $c / a=0.9546 .{ }^{23}$ Neutron powder diffraction up to $2.6 \mathrm{GPa}$ (Ref. 19) showed only a large decrease in $c / a$, but a complex structural behavior was observed later in quenched InBi alloys after thermobaric treatment $(P$ $\left.\leqslant 7 \mathrm{GPa}, T \leqslant 300^{\circ} \mathrm{C}\right) .{ }^{24} \mathrm{New}$ phases were obtained in metastable states (at $P=0 \mathrm{GPa}, T=-100^{\circ} \mathrm{C}$ ), some showing simple hexagonal structure $(h P 1)$ and others more complex structures which are not yet determined definitely, motivat- ing the present structural study on InBi in situ under pressures up to $75 \mathrm{GPa}$.

\section{EXPERIMENTAL DETAILS}

The present study was performed with diamond-anvil cells $^{25,26}$ and energy-dispersive $\mathrm{x}$-ray diffraction (EDXD) using synchrotron radiation at the beamline $F 3$ of HASYLAB/DESY. ${ }^{27,28}$ The samples were mounted in gasket holes $120 \mu \mathrm{m}$ diameter or larger, together with ruby or gold as pressure marker ${ }^{29,30}$ and mineral oil as the pressure transmitting medium. The sample material was obtained by melting stoichiometric amounts of the pure elements (99.99\%) subjected to thermobaric treatment, as described previously. ${ }^{24}$ Small pieces from these samples were used for four sets of experiments at room temperature. In two additional series the samples were heated in the diamond cell under pressure up to $240{ }^{\circ} \mathrm{C}$ for $1-2 \mathrm{~h}$ to check for special effects of kinetics, recrystallization, and order-disorder transitions. Specially adopted software ${ }^{31}$ was used for the evaluation of the diffraction spectra.

\section{RESULTS AND DISCUSSION}

The diffraction spectra for the initial phase $\operatorname{InBi}(\mathrm{I})$ were strongly effected by texture. Nevertheless, the diffraction spectra gave clear evidence for the formation of a new phase II at pressures 7-8 GPa. The positions and intensities of the corresponding diffraction peaks changed gradual after this transition up to 20-22 GPa, where systematic changes in the diffraction spectra signaled the occurrence of a new phase III. Typical diffraction spectra of InBi in the three modifications are shown in Fig. 1. To reduce effects from stress and texture, one sample was pressurized to $20 \mathrm{GPa}$ and annealed at this pressure for $2 \mathrm{~h}$ at $240{ }^{\circ} \mathrm{C}$. The spectrum obtained at $20.1 \mathrm{GPa}$ after cooling to room temperature corresponds to pure $\mathrm{InBi}(\mathrm{III})$. The spectra in Fig. 1 for 3.1 and $0 \mathrm{GPa}$ were 


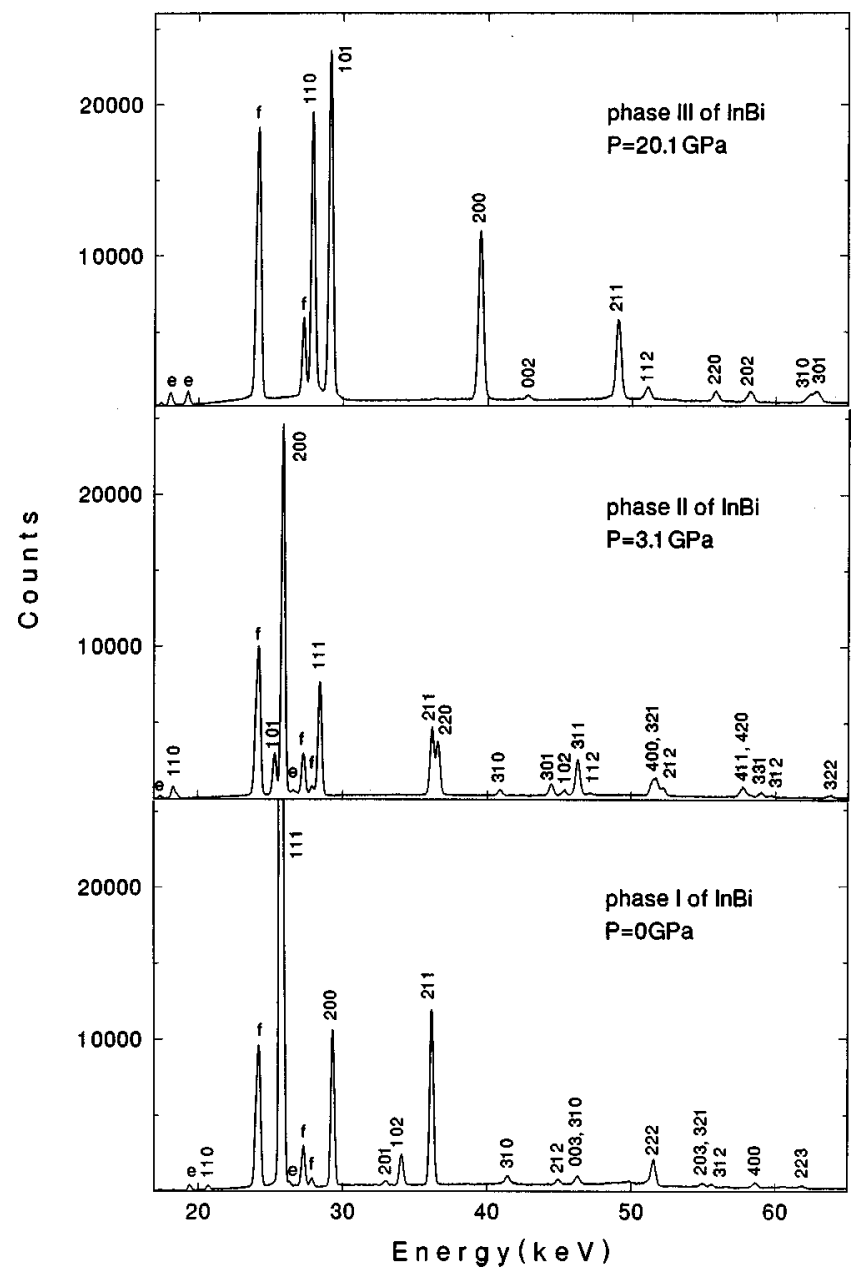

FIG. 1. EDXD spectra of InBi at different pressures measured with $\Theta=4.85^{\circ} . f$ and $e$ denote fluorescence and escape peaks, respectively. Diffraction lines of phases I and II are indexed according to $t P 4$ and phase III according to $t I 2$.

obtained from the same sample on decompression and correspond to $\operatorname{InBi}(\mathrm{II})$ and $\operatorname{InBi}(\mathrm{I})$, respectively. These spectra illustrate the complete reversibility of these two transformations.

A structural assignment for the diffraction data of $\operatorname{InBi}(\mathrm{II})$ is given in Fig. 1 and shown in more detail in Table I. Special assignment software ${ }^{31}$ finds only this one solution with four atoms in the unit cell according to a tetragonal primitive metric $t P 4$, which gives indeed a perfect matching for all the observed and calculated line positions within the resolution of the EDXD technique.

In fact, this metric represents only a sudden change in $c / a$ from $\sim 0.91$ to $\sim 0.61$ at the transition from phase I to II. If one adopts the same space group $P 4 / \mathrm{nmm}$ for both phases with In on the position $2 a(000,1 / 21 / 20)$ and $\mathrm{Bi}$ on $2 c$ $(01 / 2 z ; 1 / 20 \bar{z})$ one finds also reasonable agreement between observed and calculated intensities for the corresponding pattern at $3.1 \mathrm{GPa}$ with $z=0.33$. The decrease in the intensity ratio $I_{211} / I_{220}$ towards zero at higher pressures points to an increase in $z \rightarrow 0.5$ and the ratio $I_{211} / I_{220}>1$ at $3.1 \mathrm{GPa}$ is only compatible with ordering of In on $2 a$ and $\mathrm{Bi}$ on $2 c$ sites but not with the opposite assignment. On the other hand, for $z=1 / 2$ this structure can be represented by a smaller tetragonal unit cell with $a_{\text {new }}=a_{\text {old }} / \sqrt{2}$ with its two
TABLE I. Observed and calculated $d$ spacings and integrated intensities for the EDXD spectrum of $\operatorname{InBi}(\mathrm{II}, t P 4)$ at $3.1 \mathrm{GPa}$. The best fit gives $a=566.5(1) \mathrm{pm}$ and $c=337.3(1) \mathrm{pm}$. Intensities are calculated for $z=0.33$ and normalized to $I(200)$. A sample thickness of $40 \mu \mathrm{m}$ was assumed.

\begin{tabular}{cccrr}
\hline \hline$(h k l)$ & $d_{\text {obs }}(\mathrm{pm})$ & $d_{\text {calc }}(\mathrm{pm})$ & $I_{\text {obs }}$ & $I_{\text {calc }}$ \\
\hline 110 & 400.4 & 400.6 & 4 & 8 \\
101 & 289.7 & 289.8 & 12 & 60 \\
200 & 283.2 & 283.3 & 100 & 100 \\
111 & 258.0 & 258.0 & 32 & 62 \\
211 & 202.6 & 202.6 & 20 & 42 \\
220 & 200.4 & 200.3 & 16 & 33 \\
310 & 179.2 & 179.1 & 2 & 3 \\
301 & 164.8 & 164.8 & 4 & 8 \\
102 & 161.5 & 161.6 & 2 & 7 \\
311 & 158.3 & 158.2 & 12 & 20 \\
112 & 155.4 & 155.4 & 1 & 9 \\
321 & 142.0 & 142.4 & 10 & 8 \\
400 & 140.3 & 141.6 & & 6 \\
212 & 140.4 & 2 & 7 \\
411 & 126.9 & 127.2 & 5 & 4 \\
420 & 125.7 & 126.7 & & 6 \\
302 & 124.2 & 125.8 & 1 & 2 \\
331 & 122.8 & 122.8 & 2 & 3 \\
312 & 115.0 & 115.0 & 1 & 4 \\
322 & & & & \\
\hline \hline
\end{tabular}

atoms in the positions (000) and (1/2 1/2 1/2). The fitting of the observed diffraction peaks to this tetragonal cell is illustrated in Table II. Thereby the difference in the atomic numbers for In (49) and $\mathrm{Bi}(83)$ is large enough to cause some "difference" reflections for an ordered structure, however there was no evidence for this atomic ordering in the spectra, and therefore this new structure must be described as bodycentered tetragonal $(t I 2)$, which is the same structure as for the high-pressure form of tin.

Figure 2 represents the change of the average atomic volume vs pressure for the three phases. The equation of state (EOS) for phase III is represented equally well by two dif-

TABLE II. Observed and calculated $d$ spacings and integrated intensities for the EDXD spectrum of disordered $\operatorname{InBi}(\mathrm{III}, t I 2)$ at $20.1 \mathrm{GPa}$. The best fit gives $a=371.3(1) \mathrm{pm}$ and $c$ $=342.3(1) \mathrm{pm}$. Intensities are normalized to I(101). A sample thickness of $40 \mu \mathrm{m}$ was assumed.

\begin{tabular}{cccrr}
\hline \hline$(h k l)$ & $d_{\text {obs }}(\mathrm{pm})$ & $d_{\text {calc }}(\mathrm{pm})$ & $I_{\text {obs }}$ & $I_{\text {calc }}$ \\
\hline 110 & 262.6 & 262.5 & 72 & 63 \\
101 & 251.7 & 251.7 & 100 & 100 \\
200 & 185.7 & 185.7 & 59 & 20 \\
002 & 171.4 & 171.1 & 2 & 7 \\
211 & 149.4 & 149.4 & 29 & 30 \\
112 & 143.4 & 143.4 & 5 & 12 \\
220 & 131.3 & 131.3 & 4 & 5 \\
202 & 125.9 & 125.8 & 5 & 6 \\
310 & 117.5 & 117.4 & 3 & 4 \\
301 & 116.5 & 116.4 & 5 & 4 \\
\hline \hline
\end{tabular}




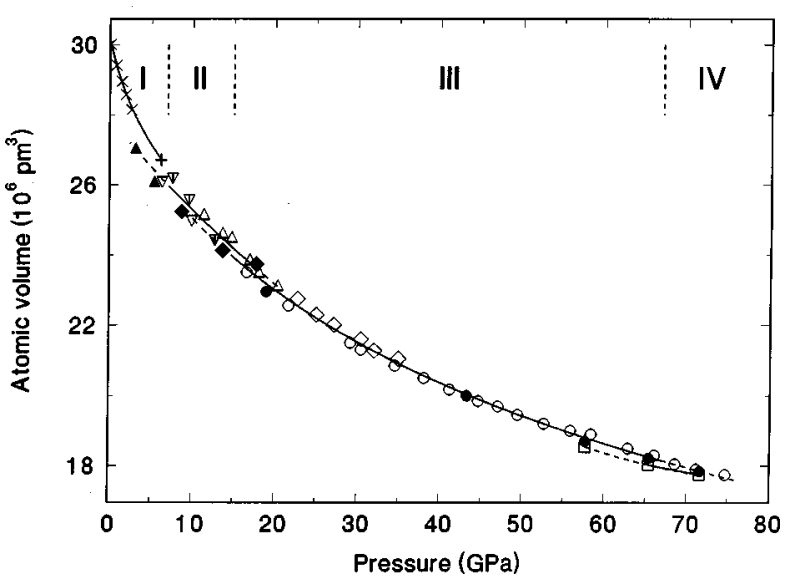

FIG. 2. Effect of pressure on the atomic volume for InBi. Crosses: phase I (Ref. 19 and one data point from the present work at $5 \mathrm{GPa}$ ). Triangles: phase II (three different runs). Diamonds and circles: phase III. Squares: phase IV. Open symbols denote compression and solid symbols decompression, respectively.

ferent EOS forms, however, with significantly different values for the commonly used free parameters: $V_{0}$ for the (extrapolated) volume at ambient pressure, $K_{0}$ for the corresponding bulk modulus, and $K_{0}^{\prime}$ for its pressure derivative. The second-order Birch equation ${ }^{32}$ results in $V_{0}$ $=28.2(4) \times 10^{6} \mathrm{pm}^{3}, \quad K_{0}=67(8) \mathrm{GPa}$, and $K_{0}^{\prime}=3.8(2)$, whereas the more recently proposed form H12 (Ref. 30) gives $V_{0}=27.98(2) \times 10^{6} \mathrm{pm}^{3}, K_{0}=74.7(3) \mathrm{GPa}$, and $K_{0}^{\prime}$ $=3.05(2)$ with significantly smaller standard deviations for the strongly correlated parameter values.

Changes in the axial ratio for the phases II and III with pressure are shown in Fig. 3, which gives also the structural relation between phases $\mathrm{II}(t P 4)$ and $\mathrm{III}(t I 2)$ assuming that phase III is disordered. With increasing pressure $c / a$ of $\operatorname{InBi}(t I 2)$ (phase III) shows at first a steeper increase and later an almost constant value of about 0.960 up to the high-

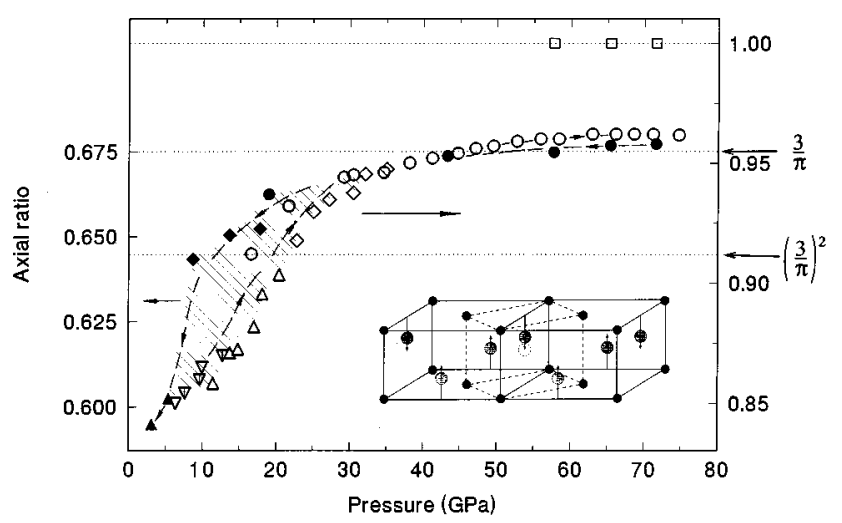

FIG. 3. $c / a$ ratios vs pressure for $\mathrm{InBi}(\mathrm{II})$ (left-hand scale) and for $\mathrm{InBi}(\mathrm{III})$ (right-hand scale). The values $3 / \pi$ and $(3 / \pi)^{2}$ represent special cases of $c / a$ (see text). $c / a=1$ corresponds to cubic $\mathrm{InBi}(\mathrm{IV})$. The shaded area represents a region of hysteresis for the II-III phase transition. The inset illustrates structural relations for the $\mathrm{InBi}(\mathrm{II})$-(III) transition. Solid lines represent the structure of $\mathrm{InBi}(\mathrm{II}, t P 4)$ with $\mathrm{In}$ in the position $2 a(000 ; 1 / 21 / 20)$ and $\mathrm{Bi}$ in $2 c(01 / 2 z ; 1 / 20 z)$. At the transition to $\operatorname{InBi}(\mathrm{III}, t I 2) z$ increases to $1 / 2$ reducing the cell to $t I 2$ (dashed lines) with disordering of the atoms.

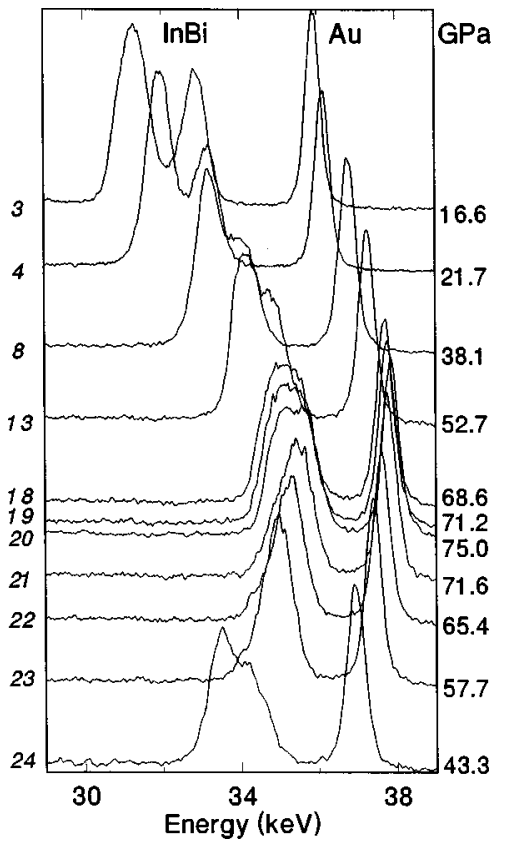

FIG. 4. Effect of pressure on InBi and Au diffraction peaks in EDXD spectra $\left(\Theta=4.24^{\circ}\right)$ showing the approach towards cubic $\mathrm{InBi}(\mathrm{IV})$ in the spectra numbered 3-23 and the reversal on further decompression.

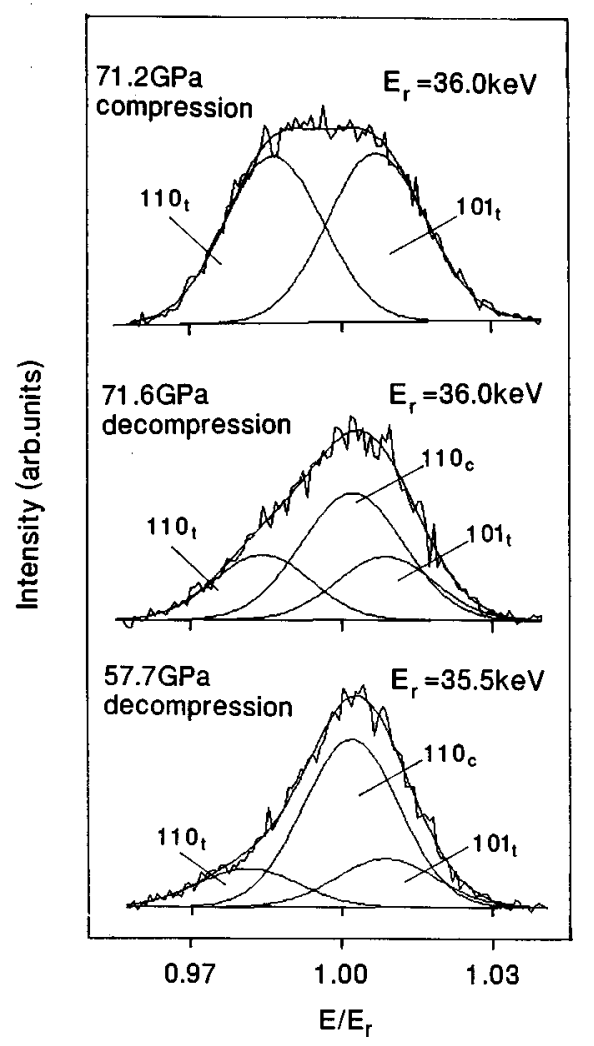

FIG. 5. Peak profiles fitted as doublet $(110+101)_{t}$ at $71.2 \mathrm{GPa}$ and as triplet including $(110)_{c}$ and $(110+101)_{t}$ at decompression. The values for the halfwidths were fixed for all three peaks to the values from well resolved spectra at lower pressures. To compensate for the line shifts, the energies are scaled to different reference values $E_{r}$. 
est pressure of $75 \mathrm{GPa}$ for the present study. However, on decompression (Fig. 4) the (110) + (101) doublet changes its shape and a deconvolution gives strong hints for the admixture of a third component (Fig. 5) corresponding to a (110) peak for a new cubic phase IV similar to the high-pressure behavior of $\mathrm{Sn}^{21}{ }^{21}$ The admixture of this extra line for a new phase $\operatorname{InBi}(c I 2)$ (phase IV) increases at first on further decompression down to about $60 \mathrm{GPa}$ (Fig. 5) before the cubic component disappears around $50 \mathrm{GPa}$. From the number of observed lines one would conclude that this new phase IV would also show disorder with respect to the occupation of the lattice sites by In or $\mathrm{Bi}$ atoms and the change in the lattice parameters in the III-IV phase transition would correspond to a volume change of less than $1 \%$ explaining to some extend the sluggishness of the transition. Certainly, this interpretation of the present spectra needs further support by measurements at higher pressures, by pressure cycling around the transition, and by measurements at higher temperatures. Since however similar features of a tetragonal-tocubic transition with small volume change, discontinuity in $c / a$, and phase coexistence over a substantial range of pressure were previously observed for tin, ${ }^{21,33}$ and theoretical high-pressure studies for $\operatorname{tin}^{34}$ indicate that the energy difference between these two phases is indeed very small over a large range in pressure, it appears necessary to point out this possible interpretation of the present spectra in the range between 60 and $80 \mathrm{GPa}$.
The tetragonal distortions for the high-pressure phases of both tin and InBi can be explained qualitatively within the nearly-free-electron approach due to the additional lowering in the crystal energy by the so-called band-structure terms. If one considers for $s p$ metals the Fermi sphere nesting in the Brillouin zone of some noncubic structures, special values in axial ratios for certain hexagonal and tetragonal structures can be stabilized for specific values of the number of free electrons per atom $n_{e} \cdot{ }^{35}$

For the $c 12$ structure with $n_{e}=4$ one finds that $k_{F}$ is larger than the lattice parameter $2 \pi / a$ of the reciprocal lattice and a distortion to $t I 2$ is favored with an optimum value of $c / a=3 / \pi=0.955$. Simple geometric relations between $k_{F}$ and reciprocal-lattice parameters of cubic and tetragonal structures, for instance $k_{F} /\left(2 \pi / a_{c}\right)=\left(2 \pi / a_{t}\right) / k_{F}$, provide another special value for the axial ratio: $c / a=(3 / \pi)^{2}$ $=0.912$. These values are very close to the experimentally observed limits of $c / a$ ratios for the $t I 2$ phases of Sn (Refs. 21, 33) and $\operatorname{InBi(III)~(see~Fig.~3).~}$

\section{ACKNOWLEDGMENTS}

The authors wish to thank G. Reiss and W. Sievers for experimental assistance. V.D. acknowledges support from DAAD.
${ }^{1}$ S. K. Sikka, H. Olijnyk, and W. B. Holzapfel, Phys. Lett. 103A, 137 (1984).

${ }^{2}$ J. Z. Hu and I. L. Spain, Solid State Commun. 51, 263 (1984).

${ }^{3}$ S. I. Duclos, Y. K. Vohra, and A. L. Ruoff, Phys. Rev. Lett. 58, 775 (1987).

${ }^{4}$ Y. K. Vohra, E. Brister, S. Desgreniers, A. L. Ruoff, K. L. Chang, and M. L. Cohen, Phys. Rev. Lett. 56, 1944 (1986).

${ }^{5}$ M. T. Yin and M. L. Cohen, Phys. Rev. B 26, 5668 (1982).

${ }^{6}$ K. J. Chang and M. L. Cohen, Phys. Rev. B 30, 5376 (1984).

${ }^{7}$ S. T. Weir, Y. K. Vohra, and A. L. Ruoff, Phys. Rev. B 36, 4543 (1987).

${ }^{8}$ C. A. Vanderborgh, Y. K. Vohra, and A. L. Ruoff, Phys. Rev. B 40, 12450 (1989).

${ }^{9}$ S. T. Weir, Y. K. Vohra, C. A. Vanderborgh, and A. L. Ruoff, Phys. Rev. B 39, 1280 (1989).

${ }^{10}$ R. J. Nelmes, M. I. McMahon, P. D. Hatton, J. Crain, and R. O. Piltz, Phys. Rev. B 47, 35 (1993).

${ }^{11}$ M. I. McMahon, R. J. Nelmes, N. G. Wright, and D. R. Allan, Phys. Rev. B 50, 13047 (1994).

${ }^{12}$ M. I. McMahon and R. J. Nelmes, J. Phys. Chem. Solids 56, 485 (1995).

${ }^{13}$ R. J. Nelmes and M. I. McMahon, Phys. Rev. Lett. 74, 106 (1995).

${ }^{14}$ S. B. Zhang and M. L. Cohen, Phys. Rev. B 35, 7604 (1987).

${ }^{15}$ S. B. Zhang and M. L. Cohen, Phys. Rev. B 39, 1450 (1989).

${ }^{16}$ G. Y. Guo, J. Crain, P. Blaha, and W. M. Temmerman, Phys. Rev. B 47, 4841 (1993).

${ }^{17}$ A. Garcia and M. L. Cohen, Phys. Rev. B 47, 6751 (1993).

${ }^{18}$ A. Mujica, R. J. Needs, and A. Munoz, Phys. Rev. B 52, 8881 (1995)

${ }^{19}$ J. D. Jorgensen and J. B. Clark, Phys. Rev. B 22, 6149 (1980).

${ }^{20}$ J. D. Barnett, V. E. Bean, and H. T. Hall, J. Appl. Phys. 37, 875
(1966).

${ }^{21}$ H. Olijnyk and W. B. Holzapfel, J. Phys. (Paris) Colloq. 45, C8153 (1984).

${ }^{22}$ S. Desgreniers, Y. K. Vohra, and A. L. Ruoff, Phys. Rev. B 39, 10359 (1989).

${ }^{23}$ Pearson's Handbook of Crystallographic Data for Intermetallic Phases, edited by P. Villars and L. D. Calvert (American Society for Metals, Metals Park, OH, 1985).

${ }^{24}$ V. F. Degtyareva, S. A. Ivakhnenko, E. G. Ponyatovskii, and V. I. Rashchupkin, Fiz. Tverd. Tela (Leningrad) 24, 1350 (1982) [Sov. Phys. Solid State 24, 770 (1982)].

${ }^{25}$ K. Syassen and W. B. Holzapfel, Europhys. Conf. Abstr. 1A, 75 (1975).

${ }^{26}$ W. B. Holzapfel, in High Pressure Chemistry, edited by H. Kelm (Reidel, Boston, 1978), p. 177.

${ }^{27}$ W. A. Grosshans, E.-F. Düsing, and W. B. Holzapfel, High Temp.-High Press. 16, 539 (1984).

${ }^{28}$ J. W. Otto, Nucl. Instrum. Methods Phys. Res. A 384, 552 (1997).

${ }^{29}$ H. K. Mao, P. M. Bell, J. W. Shaner, and D. J. Steinberg, J. Appl. Phys. 49, 32 (1978).

${ }^{30}$ W. B. Holzapfel, Europhys. Lett. 16, 67 (1991); Rep. Prog. Phys. 59, 29 (1996).

${ }^{31}$ F. Porsch, EDXPOWDER-program for evaluation of EDXD spectra, RTI, Paderborn, Germany, 1969.

${ }^{32}$ F. Birch, J. Geophys. Res. 83, 1257 (1978).

${ }^{33}$ M. Liu and L. Liu, High Temp.-High Press. 18, 79 (1986).

${ }^{34}$ J. L. Corkill, A. Garcia, and M. L. Cohen, Phys. Rev. B 43, 9251 (1991).

${ }^{35}$ V. F. Degtyareva, in Stability of Materials, Vol. 355 of NATO Advanced Study Institute, Series B: Physics, edited by A. Gonis, P. E. A. Turchi, and J. Kudrnovsky (Plenum, New York, 1996), p. 465. 